Pacific Journal of Mathematics

ON CONTINUOUS MATRIX-VALUED FUNCTIONS ON A 


\section{ON CONTINUOUS MATRIX-VALUED FUNCTIONS ON A STONIAN SPACE}

\section{Don Deckard and Carl Pearcy}

1. Introduction. In this paper the authors continue the study (begun in [9] and carried on in [3] and [10]) of matrices with entries from the algebra $C(\mathfrak{X})$ of all continuous complex-valued functions on an extremely disconnected, compact Hausdorff space $\mathfrak{X}$. (Such spaces are sometimes called Stonian after M. H. Stone, who considered them in [14].) One of the authors has shown ([10], Theorem 3) that if $A$ and $B$ are $n \times n$ matrices over $C(\mathfrak{X})$ such that $A(x)$ is unitarily equivalent to $B(x)$ for each $x \in \mathfrak{X}$, then $A$ and $B$ are unitarily equivalent in the algebra $M_{n}(\mathfrak{X})$ of all $n \times n$ matrices over $C(\mathfrak{X})$. It is thus natural to ask whether the similarity of $A(x)$ and $B(x)$ for each $x \in \mathfrak{X}$ is sufficient to guarantee the similarity of $A$ and $B$ in $M_{n}(\mathfrak{X})$. We show by example in $\S 2$ that the answer is no; however, we also show that if the hypothesis is strengthened by the addition of a uniform boundedness requirement, then the similarity of $A$ and $B$ in $M_{n}(\mathfrak{X})$ does indeed follow. As a by-product of the technique introduced to give this result, we obtain a new short proof of Theorem 3 of [10].

In $\S 3$ we show that a certain class of entire functions maps $M_{n}(\mathfrak{X})$ onto itself; this is a generalization (with a different proof) of a result of Kurepa [8] for $n \times n$ matrices, and adds to the information obtained by Brown [1] on the question of which entire functions map which Banach algebras onto themselves. As a corollary, we learn that every invertible element of $M_{n}(\mathfrak{X})$ has a logarithm. Section 4 is devoted to proving that an element of $M_{n}(\mathfrak{X})$ has an identically vanishing trace if and only if it is a commutator in $M_{n}(\mathfrak{X})$. (See Remark 2, $\S 4$, for a paraphrase of this result cast in the terminology of operator theory on Hilbert space.) Finally, in $\S 5$ the authors give two examples which indicate that it is probably fruitless to pursue the structure theory of matrices over $C(\mathfrak{X})$ where $\mathfrak{X}$ is a more general topological space than a Stonian space.

2. Similarity in $M_{n}(\mathfrak{X})$. The most convenient definition of $M_{n}(\mathfrak{X})$ is as follows. Let $M_{n}$ denote the full ring of $n \times n$ complex matrices under the operator norm, and let $\mathfrak{X}$ be any Stonian space. Denote by $M_{n}(\mathfrak{X})$ the *-algebra of continuous functions from $\mathfrak{X}$ to $M_{n}$, where the algebraic operations in $M_{n}(\mathfrak{X})$ are defined pointwise. Under the norm $\|A\|=\sup _{x \in \mathfrak{X}}\|A(x)\|, M_{n}(\mathfrak{X})$ is a $C^{*}$-algebra identifiable with the $C^{*}$ algebra of all $n \times n$ matrices over $C(\mathfrak{X})$. Moreover, $M_{n}(\mathfrak{X})$ is an

Received July 12, 1963. 
$A W^{*}$-algebra [7], and this fact is used briefly in this section.

We first show that pointwise similarity of $A(x)$ and $B(x)$ on $\mathfrak{X}$ is not sufficient to ensure that $A$ and $B$ be similar in $M_{n}(\mathfrak{X})$. For this purpose, let $\mathscr{S}$ be the Stone-Czech compactification of the natural numbers $\mathscr{N}$. Then $\mathscr{S}$ is a Stonian space. (See, for example, the discussion on page 295 of [12].) Consider elements $A$ and $B$ of $M_{2}(\mathscr{S})$ defined by:

$$
A(x)=\left(\begin{array}{ll}
0 & 1 / x^{2} \\
0 & 0
\end{array}\right), \quad B(x)=\left(\begin{array}{ll}
0 & 1 / x \\
0 & 0
\end{array}\right)
$$

for each natural number $x \in \mathscr{N}$. Then $A(x)=B(x)=0$ for $x \in \mathscr{S}-\mathscr{N}$, and it is obvious that $A(x)$ and $B(x)$ are similar for each $x \in \mathscr{S}$. Suppose that $S=\left(s_{i j}\right)$ is an invertible element in $M_{2}(\mathscr{S})$ satisfying $S A=B S$. Calculation yields $s_{21}(x)=0$ for $x \in \mathscr{N}$ so that $s_{21} \equiv 0$. Furthermore, $s_{11}(x)=x s_{22}(x)$ for $x \in \mathscr{N}$, and the invertibility of $S$ guarantees that $s_{22}$ never vanishes. Thus $s_{11}$ is unbounded, contradicting $s_{11} \in C(\mathscr{S})$, and it follows that $A$ and $B$ are not similar in $M_{2}(\mathscr{S})$.

The following theorem gives necessary and sufficient conditions for $A$ and $B$ to be similar in $M_{n}(\mathfrak{X})$.

Theorem 1. Let $\mathfrak{X}$ be any Stonian space, and let $A, B \in M_{n}(\mathfrak{X})$. Suppose that there is a dense subset $\mathscr{D} \subset \mathfrak{X}$ and a positive number $M$ such that for $x \in \mathscr{D}$, there is an invertible matrix $S(x)$ satisfying $S(x) A(x) S^{-1}(x)=B(x),\|S(x)\|<M$, and $\left\|S^{-1}(x)\right\|<M$. Then there is an invertible element $T \in M_{n}(\mathfrak{X})$ satisfying $T A T^{-1}=B,\|T\| \leqq M$, and $\left\|T^{-1}\right\| \leqq M$.

Proof. We consider collections $\left\{\mathscr{U}_{i}\right\}$ of nonempty, disjoint, compact open sets $\mathscr{U}_{i} \subset \mathfrak{X}$ with the property that if $\mathscr{U}_{i} \in\left\{\mathscr{U}_{i}\right\}$, then there is an invertible element $T_{i} \in M_{n}\left(\mathscr{C}_{i}\right)$ satisfying $T_{i}(x) A(x) T_{i}^{-1}(x)=B(x)$, $\left\|T_{i}(x)\right\|<M$, and $\left\|T_{i}^{-1}(x)\right\|<M$ for each $x \in \mathscr{Q}_{i}$. Let $\left\{\mathscr{U}_{i}\right\}_{i \in I}$ be a maximal such collection, and denote $\mathscr{U}=\overline{\bigcup_{i \in I} \mathscr{U}_{i}}$. Then $\mathscr{U}$ is compact open, and it follows from Lemma 2.1 of [3] that the function $\widetilde{T}$ defined on $\bigcup_{i \in I} \mathscr{C}_{i}$ so as to extend each of the $T_{i}$ can be extended to an element $T \in M_{n}(\mathscr{U})$. Similarly, there is a function $Z \in M_{n}(\mathscr{U})$ which extends each of the $T_{i}^{-1}$. It is clear from continuity considerations that $Z=T^{-1}$, and that $T$ has all the desired properties on $\mathscr{U}$, so that it suffices to prove $\dddot{\mathscr{C}}=\mathfrak{X}$. Suppose, to the contrary, that $\mathfrak{X}-\mathscr{\mathscr { C }} \neq \phi$. To obtain a contradiction, it suffices to find a compact open set $\mathscr{Y} \subset \mathfrak{X}-\mathscr{C}$ and an invertible element $V \in M_{n}\left(\mathscr{Y}^{\prime}\right)$ such that for $x \in \mathscr{Y}$, $V(x) A(x)=B(x) V(x),\|V(x)\|<M$, and $\left\|V^{-1}(x)\right\|<M$. To do this, we regard the equation $V A=B V$ as a system of linear equations 


$$
\begin{aligned}
& c_{11} v_{1}+c_{12} v_{2}+\cdots+c_{1 m} v_{m}=0 \\
& \cdots \cdots \cdots \cdots \cdots+\cdots \cdots+\cdots \cdots+\cdots \cdots+c_{m m} v_{m}=0 \\
& c_{m 1} v_{1}+c_{m 2} v_{2}+\cdots+
\end{aligned}
$$

where

(1) the unknown functions $v_{i}$ are the entries, in some prescribed order, of the matrix $V$

(2) the coefficients $c_{i j} \in C(\mathfrak{X}-\mathscr{C})$ are the appropriate combinations of the entries of the matrices $A$ and $B$

(3) $m=n^{2}$.

For $x \in \mathfrak{X}-\mathscr{U}$, consider the corresponding system $(L(x))$ of linear equations, and let $x_{0} \in \mathfrak{X}-\mathscr{Z}$ be a point such that the rank $r(x)$ of the system $(L(x))$ assumes its maximum $r_{0}$ at $x_{0}$. (The case $r_{0}=0$ leads trivially to a contradiction of $\mathfrak{X}-\mathscr{U} \neq \phi$, and we ignore it. The case $r_{0}=m$ cannot occur.) Then there is some $r_{0} \times r_{0}$ minor $N$ of the coefficient determinant of the system $\left(L\left(x_{0}\right)\right)$ which is nonzero, and by continuity there exists a compact open neighborhood $\mathscr{Y} \subset \mathfrak{X}-\mathscr{U}$ of $x_{0}$ such that for $x \in \mathscr{V} 1$, the same minor $N$ remains a nonzero minor of maximum size. According to the hypothesis, there is a point $x_{1} \in \mathscr{Y} /$ and an invertible matrix $S\left(x_{1}\right)$ such that $S\left(x_{1}\right) A\left(x_{1}\right)=B\left(x_{1}\right) S\left(x_{1}\right),\left\|S\left(x_{1}\right)\right\|<M$, and $\left\|S^{-1}\left(x_{1}\right)\right\|<M$. Let the corresponding nontrivial solution of the system $\left(L\left(x_{1}\right)\right)$ be denoted by $\left(\mu_{1}, \mu_{2}, \cdots, \mu_{m}\right)$ (i.e., the $\mu_{i}$ are the entries of the matrix $\left.S\left(x_{1}\right)\right)$. We wish to define an $m$-tuple $\left(v_{1}(x), v_{2}(x), \cdots, v_{m}(x)\right)$ at each point of $\mathscr{Y} 1$ in such a way that

(1) the $m$-tuple is a solution of $(L(x))$ for each $x \in \mathscr{Y}$,

(2) $v_{i} \in C\left(\mathscr{V}_{1}\right)$ for $1 \leqq i \leqq m$, and

(3) $v_{i}\left(x_{1}\right)=\mu_{i}$ for $1 \leqq i \leqq m$. This is accomplished as follows. Since for $x \in \mathscr{V}, N$ is a nonzero minor of maximum size, it suffices to solve (continuously on $\mathscr{V}_{1}$ ) the $r_{0}$ equations affiliated with $N$. Thus for the appropriate $m-r_{0}$ values of $i$ (the values not affiliated with $N)$, define $v_{i}(x) \equiv \mu_{i}$ on $\mathscr{V}_{1}$; then for $x \in \mathscr{V}_{1}$ the other $r_{0}$ numbers $v_{i}(x)$ are determined by Cramer's rule, and since the functions $c_{i j}$ are continuous it follows that (1), (2), and (3) above are satisfied. Next place the resulting functions $v_{i} \in C\left(\mathscr{V}_{1}\right)$ in their appropriate positions in the matrix $V$, and shrink the neighborhood $\mathscr{V}_{1}$ of $x_{1}$ to a compact open neighborhood $\mathscr{Y} \subset \mathscr{Y}$ of $x_{1}$ such that for $x \in \mathscr{Y}$, the matrix $V(x)$ is invertible and the inequalities $\|V(x)\|<M$ and $\left\|V^{-1}(x)\right\|<M$ remain valid. The existence of the compact open set $\mathscr{Y}$ contradicts the maximality of the collection $\left\{\mathscr{C}_{i}\right\}_{i \in I}$, and thus the proof is complete.

We can prove Theorem 3 of [10] in a similar fashion, 
THEOREM 2. If $\mathfrak{X}$ is Stonian and $A, B \in M_{n}(\mathfrak{X})$ are such that $A(x)$ and $B(x)$ are unitarily equivalent at each point of a dense subset of $\mathfrak{X}$, then $A$ and $B$ are unitarily equivalent in $M_{n}(\mathfrak{X})$.

Proof. We consider collections $\left\{\mathscr{C}_{i}\right\}$ of nonempty, disjoint, compact open subsets $\mathscr{U}_{i} \subset \mathfrak{X}$ with the property that if $\mathscr{U}_{i} \in\left\{\mathscr{U}_{i}\right\}$, then there is a unitary element $U_{i} \in M_{n}\left(\mathscr{U}_{i}\right)$ satisfying $U_{i}(x) A(x) U_{i}^{*}(x)=B(x)$ for each $x \in \mathscr{U}_{i}$. As before, we choose a maximal collection $\left\{\mathscr{U}_{i}\right\}_{i \in I}$, and define $\mathscr{U}=\overline{\bigcup_{i \in I} \mathscr{U}_{i}}$. Again it suffices to prove $\mathscr{U}=\mathfrak{X}$. The argument then proceeds exactly as above, except that the system of linear equations to be considered is the system equivalent to the pair of equations $V A=B V$ and $V A^{*}=B^{*} V$. (Thus the system consists of $2 n^{2}$ equations in $n^{2}$ unknowns, but it is clear that this has no effect on the argument.) Then, proceeding essentially as above, we obtain a compact open subset $\mathscr{Y} \subset \mathfrak{X}-\mathscr{U}$ and an invertible (not necessarily unitary) element $V \in M_{n}\left(\mathscr{Y}^{-}\right)$ such that for $x \in \mathscr{Y}, V(x) A(x)=B(x) V(x)$ and $V(x) A^{*}(x)=B^{*}(x) V(x)$. One knows from ([14], Lemma 2.1) that we can write $V$ in polar form $V=U P$ where $U$ is a unitary element of $M_{n}(\mathscr{V})$. A standard calculation shows that for $x \in \mathscr{Y}, U(x) A(x) U^{*}(x)=B(x)$; thus the existence of $\mathscr{Y}^{-}$contradicts the maximality of the collection $\left\{\mathscr{U}_{i}\right\}_{i \in I}$, and the proof is complete.

REMARK. One would naturally like to have a collections of global objects to attach to an element $A \in M_{n}(\mathfrak{X})$ which would serve as a complete set of similarity invariants for $A$. In this connection, it is easy to see that one cannot always obtain an element $J \in M_{n}(\mathfrak{X})$ such that $A$ is similar to $J$ in $M_{n}(\mathfrak{X})$ and such that $J(x)$ is in Jordan form for each $x \in \mathfrak{X}$.

3. Entire functions on $M_{n}(\mathfrak{X})$. We say that an entire function $f$ has property $(K)$ if, for every complex number $\zeta$, there is a complex number $z$ satisfying $f(z)=\zeta$ and $f^{\prime}(z) \neq 0$. In [8] Kurepa showed that an entire function $f$ maps $M_{n}$ onto itself if and only if $f$ has property $(K)$. The study was then taken up by Brown [1] who characterized the class of entire functions $f$ which map the algebra $\mathscr{L}(\mathscr{H})$ of all bounded operators on an infinite dimensional Hilbert space $\mathscr{H}$ onto itself. Brown showed that such an $f$ maps every Banach algebra onto itself, and we say that such an $f$ has property $(B)$. Since certain $W^{*}$-algebras of operators on Hilbert space have faithful $C^{*}$-representations as an $M_{n}(\mathfrak{X})$ (see [9]), one has, in a sense, $\mathscr{L}(\mathscr{H}) \supset M_{n}(\mathfrak{X}) \supset M_{n}$. Thus it is of interest to discover which entire functions map $M_{n}(\mathfrak{X})$ onto itself, and the answer is given by

THEOREM 3. If $f$ is an entire function and $\mathfrak{X}$ is a Stonian space, then $f$ maps $M_{n}(\mathfrak{X})$ onto itself if and only if $f$ has property $(K)$. 
Proof. Since for each $x \in \mathfrak{X},[p(A)](x)=p(A(x))$ for every polynomial $p(z)$, and since $f$ is the uniform limit of polynomials on compact sets of the z-plane, $[f(A)](x)=f(A(x))$ for each $x \in \mathfrak{X}$. Thus, if $f$ maps $M_{n}(\mathfrak{X})$ onto itself, then $f$ must map $M_{n}$ onto itself, so that by Kurepa's theorem [8], $f$ has property $(K)$. Now suppose that $f$ has property $(K)$, and let $A \in M_{n}(\mathfrak{X})$. We look for $B \in M_{n}(\mathfrak{X})$ such that $f(B) \quad A$. Let $x_{0}$ be an arbitrary point of $\mathfrak{X}$ and let $\zeta_{1}, \cdots, \zeta_{p}$ be the distinct eigenvalues of $A\left(x_{0}\right)$. Choose $z_{1}, \cdots, z_{p}$ to be complex numbers with the properties that $f\left(z_{i}\right)=\zeta_{i}$ and $f^{\prime}\left(z_{i}\right) \neq 0$. For $i=1, \cdots, p$, let $\mathscr{D}_{i}$ be a (non-degenerate) closed disc about $z_{i}$ such that $f$ is Schlicht on $\mathscr{D}_{i}$, and arrange it so that the sets $f\left(\mathscr{D}_{i}\right)$ are mutually disjoint. Let $g$ denote the inverse of the restriction of $f$ to $\bigcup_{i=1}^{p} \mathscr{D}_{i}$. Then $g$ is defined and continuous on $\mathscr{D}=\bigcup_{i=1}^{p} f\left(\mathscr{D}_{i}\right)$ and is analytic at each interior point of $\mathscr{D}$. It follows from Lemma 2.2 of [3] that there exists a compact open neighborhood $\mathscr{N}_{0}=\mathscr{N}^{\prime}\left(x_{0}\right)$ of $x_{0}$ such that for $x \in \mathscr{N}_{0}$, the spectrum of $A(x)$ (denoted hereafter $\Lambda[A(x)]$ ) is a subset of the interior of $\mathscr{D}$. If $A_{0}$ denotes the restriction of $A$ to $\mathscr{N}_{0}$, then $A_{0}$ is an element of the $C^{*}$-algebra $\cdot M_{n}\left(\mathscr{N}_{0}\right)$, and it is clear that the spectrum of $A_{0}$ is $\mathrm{U}_{x \in \mathcal{N}_{0}} \Lambda[A(x)]$. As usual, following Dunford [5], $g\left(A_{0}\right) \in M_{n}\left(\mathscr{N}_{0}\right)$ can be defined as the sum of the $p$ integrals $1 / 2 \pi i \int_{\Gamma_{i}} g(\lambda)\left(A_{0}-\lambda I\right)^{-1} d \lambda$, where $\Gamma_{i}$ is the boundary of the set $f\left(\mathscr{D}_{i}\right)$. If we denote $B_{0}=g\left(A_{0}\right)$, it follows from Theorem 2.10 of [5] that $f\left(B_{0}\right)=A_{0}$. Since this construction was carried out about an arbitrary point $x_{0} \in \mathfrak{X}$, we can apply the compactness of $\mathfrak{X}$ to obtain points $x_{1}, \cdots, x_{r} \in \mathfrak{X}$ and compact open neighborhoods $\mathscr{N}_{i}$ of the $x_{i}$ such that $\bigcup_{i=1}^{r} \mathscr{N}_{i}=\mathfrak{X}$ and such that the above construction has been carried out to yield a corresponding $B_{i}$ on each $\mathscr{N}_{i}$. Furthermore, we can assume that the $\mathscr{N}_{i}$ are pairwise disjoint. The element $B \in M_{n}(\mathfrak{X})$ defined by $B(x)=B_{\imath}(x)$ for $x \in \mathscr{N}_{i}$ is such that $f(B)=A$, and the proof is complete.

CoRollaRY 3.1. If $\mathfrak{X}$ is a totally disconnected, compact Hausdorff space, then each invertible element of $M_{n}(\mathfrak{X})$ has a logarithm in $M_{n}(\mathfrak{X})$, and thus has roots of all orders in $M_{n}(\mathfrak{X})$.

Proof. Observe first that the proof of Theorem 3 above goes through word for word in the case that $\mathfrak{X}$ is only compact Hausdorff and totally disconnected. Then observe that if $A \in M_{n}(\mathfrak{X})$ and an entire function $f$ are given, in order to carry out the construction in the above proof to obtain a $B$ such that $f(B)=A$, it suffices to know that for each $\zeta$ in the spectrum of $A$, there is a complex number $z$ such that $f(z)=\zeta$ and $f^{\prime}(z) \neq 0$. These observations complete the proof.

It results easily from Theorem 3 that if 


$$
\mathfrak{A}=\sum_{k=0}^{k_{0}} \oplus M_{n_{k}}\left(\mathfrak{X}_{k}\right)
$$

is any finite $C^{*}$-sum of algebras $M_{n_{k}}\left(\mathfrak{X}_{k}\right)$ where the $\mathfrak{X}_{k}$ are Stonian spaces, then the entire functions which map $\mathfrak{A}$ onto itself are exactly those with property $(K)$. However, if one considers algebras

$$
\mathfrak{B}=\sum_{k=1}^{\infty} \oplus M_{n_{k}}\left(\mathfrak{X}_{k}\right)
$$

which are $C^{*}$-sums of infinitely many $M_{n_{k}}\left(\mathfrak{X}_{k}\right)$ where $n_{k} \rightarrow \infty$ and the $\mathfrak{X}_{k}$ are only assumed to be compact Hausdorff spaces, then the situation is different, as is demonstrated by the following theorem.

THEOREM 4. If $\mathfrak{B}$ is any algebra of the form

$$
\mathfrak{B}=\sum_{k=1}^{\infty} \oplus M_{n_{k}}\left(\mathfrak{X}_{k}\right)
$$

where $n_{k} \rightarrow \infty$ and each $\mathfrak{X}_{k}$ is a compact Hausdorff space, then the entire functions which map $\mathfrak{B}$ onto itself are exactly those with property $(B)$

The proof of this theorem is patterned after an argument of Brown [1], and depends on the following lemma.

LEMmA 3.2. Let $f$ be any entire function, let $g(z)$ be the polynomial

$$
g(z)=\sum_{i=0}^{n-1} a_{i} z^{i}
$$

and let $A \in M_{n}$ be the "analytic Toeplitz" matrix

$$
A=\left[\begin{array}{lllllll}
a_{0} & & & & & \\
a_{1} & a_{0} & & & & \\
a_{2} & a_{1} & a_{0} & & & \\
\cdot & \cdot & \cdot & \cdot & & \\
\cdot & \cdot & \cdot & \cdot & \cdot & \\
a_{n-1} & \cdot & \cdot & \cdot & a_{1} & a_{0}
\end{array}\right]
$$

Then $f(A)$ is an "analytic Toeplitz" matrix

$$
f(A)=\left[\begin{array}{ccccccc}
b_{0} & & & & & \\
b_{1} & b_{0} & & & & \\
b_{2} & b_{1} & b_{0} & & & \\
\cdot & \cdot & \cdot & \cdot & & \\
\cdot & \cdot & \cdot & \cdot & \cdot & \\
b_{n-1} & \cdot & \cdot & \cdot & b_{1} & b_{0}
\end{array}\right]
$$


and the entire function $h(z)=f(g(z))$ has a power series expansion

$$
h(z)=\sum_{i=0}^{\infty} \beta_{i} z^{i}
$$

where $\beta_{i}=b_{i}$ for $0 \leqq i \leqq n-1$.

Proof. If $f$ is any positive integral power of $z$, or more generally any polynomial, an inductive computation shows that the result is valid. For an arbitrary entire function $f$, let $p_{n}(z)$ be a sequence of polynomials which converges uniformly to $f$ on every compact subset of the $z$-plane. Then, since $p_{n}(g(z))$ converges uniformly to $h(z)$ on compact subsets of the plane, the coefficients in the power series expansions of the $p_{n}(g(z))$ must converge to the corresponding coefficients in the power series expansion of $h(z)$. (See, for example, ([2], § 211)) Furthermore, since $p_{n}(A)$ converges to $f(A)$ in the norm topology of $M_{n}$, the entries of $p_{n}(A)$ must converge to the corresponding entires of $f(A)$, and the result follows.

Proof of Theorem 4. For convenience we take $n_{k}=n$. It will be clear that this does not affect the argument. Let

$$
B=\left(\sum_{n=1}^{\infty} \oplus B_{n}\right) \in \mathfrak{B}
$$

be defined by setting

$$
B_{n} \equiv\left(\begin{array}{ccccccc}
0 & & & & & \\
1 & 0 & & & & \\
& 1 & 0 & & & \\
& & \cdot & \cdot & & \\
& & & \cdot & \cdot & \\
& & & & 1 & 0
\end{array}\right)
$$

for each positive integer $n$. Let $f$ be an entire function which maps onto $\mathfrak{B}$, and suppose that

$$
A=\sum_{n} \oplus A_{n}
$$

satisfies $f(A)=r B$ where $r$ is some fixed positive real number. Since for any central projection $E \in \mathfrak{B}, f(E A)=E f(A)$, it is clear that for each positive integer $n, f\left(A_{n}\right)=r B_{n}$. Now choose an arbitrary $x_{n} \in \mathfrak{X}_{n}$ for each integer $n$. The fact that $f\left[A_{n}\left(x_{n}\right)\right]=r B_{n}\left(x_{n}\right)$ follows just as in the proof of Theorem 3. Since $A_{n}\left(x_{n}\right)$ commutes with $B_{n}\left(x_{n}\right)=$ $1 / r f\left[A_{n}\left(x_{n}\right)\right]$ and $B_{n}$ is identically constant on $\mathfrak{X}_{n}$, a matrix calculation shows that for each positive integer $n$, the matrix $A_{n}\left(x_{n}\right)$ has the form 


$$
A_{n}\left(x_{n}\right)=\left[\begin{array}{ccccccc}
a_{0}^{n} & & & & & \\
a_{1}^{n} & a_{0}^{n} & & & & \\
a_{2}^{n} & a_{1}^{n} & a_{0}^{n} & & & \\
\cdot & \cdot & \cdot & \cdot & & \\
\cdot & \cdot & \cdot & \cdot & \cdot & \\
a_{n-1}^{n} & \cdot & \cdot & \cdot & a_{1}^{n} & a_{0}^{n}
\end{array}\right]
$$

where the $a_{i}^{n}$ are of course complex numbers. Define the sequence $g_{n}(z)$ of polynomials by

$$
g_{n}(z)=\sum_{i=0}^{n-1} a_{i}^{n} z^{i}
$$

and let $h_{n}(z)=f\left(g_{n}(z)\right)$. Since $f\left[A_{n}\left(x_{n}\right)\right]=r B_{n}\left(x_{n}\right)$, it follows from Lemma 3.2 that for each positive integer $n, h_{n}(z)$ is an entire function having a power series expansion

$$
h_{n}(z)=r z+\sum_{k=n}^{\infty} \beta_{k}^{n} z^{k} .
$$

Since $A=\sum_{n} \oplus A_{n}$ is a bounded operator, it follows that there exists a positive number $M$ such that

$$
\sum_{i=0}^{n-1}\left|a_{i}^{n}\right|^{2}<M
$$

for each $n$. Let $\mathscr{D}$ denote the disc $\mathscr{D}=\{z:|z| \leqq 1 / 2\}$ and observe that it follows from the above inequality that the sequence $g_{n}(z)$ is uniformly bounded on $\mathscr{O}$ by the number $2 \sqrt{M}$. It follows from Montel's theorem ([2], §416) that one can extract a subsequence $g_{n_{k}}(z)$ which converges uniformly on $\mathscr{D}$ to a function $g(z)$ which is analytic on $\mathscr{D}$. It follows that $h_{n_{k}}(z)=f\left(g_{n_{k}}(z)\right)$ converges uniformly to $f(g(z))$ on $\mathscr{D}$, and by virtue of the form of the power series expansion of each $h_{n_{k}}(z)$, we must have $f(g(z))=r z$ on $\mathscr{D}$. It is now clear that $g(z)$ is a Schlicht mapping of the interior $\mathscr{D}^{\circ}$ of $\mathscr{D}$ onto some bounded domain $g\left(\mathscr{D}^{\circ}\right)$ and that $f$ is a Schlicht mapping of $g\left(\mathscr{D}^{\circ}\right)$ onto the open disc $\{z:|z|<r / 2\}$. Since $r$ was arbitrary, it follows from ([1], Theorem 2) that $f$ has property $(B)$, and the proof is complete.

4. Commutators in $M_{n}(\mathfrak{X})$. We introduce the notation $\sigma(B)$ for the trace in the usual sense of an $n \times n$ complex matrix $B$. In this section, we generalize another result known for $M_{n}$, and thereby set forth a class of operators on Hilbert space which are commutators. (See Remark 2 at the end of this section.) More precisely, we establish

Theorem 5. If $\mathfrak{X}$ is a Stonian space and $A \in M_{n}(\mathfrak{X})$, then $A$ 
satisfies $\sigma[A(x)] \equiv 0$ if and only if there are elements $B$ and $C$ in $M_{n}(\mathfrak{X})$ such that $A=B C-C B$.

One half of the theorem is trivial; to prove the other half we use an idea suggested by Halmos in [6]. The crucial lemma is the following.

Lemma 4.1. If $\mathfrak{X}$ is any Stonian space and $A \in M_{n}(\mathfrak{X})$ is such that $\sigma[A(x)] \equiv 0$, then there is an invertible $S \in M_{n}(\mathfrak{X})$ such that $S A S^{-1}=D=\left(d_{i j}\right)$ satisfies $d_{11} \equiv 0$.

Proof. We consider collections $\left\{\mathscr{C}_{i}\right\}$ of disjoint, nonempty, compact open sets $\mathscr{C}_{i} \in \mathfrak{X}$ with the property that if $\mathscr{C}_{i} \in\left\{\mathscr{C}_{i}\right\}$, then there is an invertible $S_{i} \in M_{n}\left(\mathscr{U}_{i}\right)$ such that $\left\|S_{i}\right\|,\left\|S_{i}^{-1}\right\| \leqq 6$ and such that for each $x \in \mathscr{U}_{i}$, the matrix $S_{i} A S_{\imath}^{-1}(x)$ has a zero in the upper left hand corner. Let $\left\{\mathscr{C}_{i}\right\}_{i \in I}$ be a maximal such collection, and define $\mathscr{Z}=$ $\overline{U_{i \in I} \mathscr{Q}_{i}}$. It follows from Lemma 2.1 of [3] that to complete the proof, it suffices to establish $\mathscr{U}=\mathfrak{X}$. Thus, suppose to the contrary that $\mathfrak{X}-\mathscr{U} \neq \phi$. According to Theorem 1 of [3] there exist functions $\lambda_{1}, \cdots, \lambda_{n} \in C(\mathfrak{X}-\mathscr{U})$ such that for $x \in \mathfrak{X}-\mathscr{U}$, the numbers $\lambda_{1}(x), \cdots, \lambda_{n}(x)$ are exactly the eigenvalues of $A(x)$. Furthermore, there must be at least one point $x_{0} \in \mathfrak{X}-\mathscr{U}$ such that some $\lambda_{i}\left(x_{0}\right) \neq 0$. (Otherwise, we could apply Theorem 2 of [3] to obtain a unitary $U \in M_{n}(\mathfrak{X}-\mathscr{C})$ such that $U A U^{*}(x)$ is in upper triangular form for each $x \in \mathfrak{X}-\mathscr{U}$. Then the diagonal entries of $U A U^{*}(x)$ would be identically zero, and the maximality of the collection $\left\{\mathscr{U}_{i}\right\}_{i \in I}$ would be contradicted.) Since we know from the hypothesis that

$$
\sum_{i=1}^{n} \lambda_{i} \equiv 0
$$

there must be at least two distinct $i$ such that $\lambda_{i}\left(x_{0}\right) \neq 0$. In fact, a little thought convinces one that there exist $\lambda_{j}$ and $\lambda_{k}(j \neq k)$ such that

$$
0<\left|\lambda_{j}\left(x_{0}\right)\right| \leqq\left|\lambda_{k}\left(x_{0}\right)\right|<\left|\lambda_{k}\left(x_{0}\right)-\lambda_{j}\left(x_{0}\right)\right| .
$$

It follows from the circle of ideas connected with the proof of Theorem 2 of [3] that there is a unitary element $\left.U \in M_{n}(\mathfrak{X})-\mathscr{C}\right)$ such that $U A U^{*}(x)=\left(a_{i j}(x)\right)$ is in upper triangular form for each $x \in \mathfrak{X}-\mathscr{U}$ and such that $a_{11} \equiv \lambda_{k}$ and $a_{22} \equiv \lambda_{j}$ on $\mathfrak{X}-\mathscr{\mathscr { C }}$. Thus $0<\left|a_{22}\left(x_{0}\right)\right| \leqq$ $\left|a_{11}\left(x_{0}\right)\right|<\left|a_{11}\left(x_{0}\right)-a_{22}\left(x_{0}\right)\right|$, and by clever choice of $U$ (i.e., by applying an additional rotation, and then changing notation) one can arrange things so that $\left|a_{11}\left(x_{0}\right)-a_{22}\left(x_{0}\right)\right|<\left|a_{12}\left(x_{0}\right)-\left[a_{11}\left(x_{0}\right)-a_{22}\left(x_{0}\right)\right]\right|$. It follows that for some $\delta, 0<\delta<1$, there is a compact open neighborhood $\mathscr{V} \subset \mathfrak{X}-\mathscr{Z}$ of $x_{0}$ such that for $x \in \mathscr{Y}, 0<\left|a_{22}(x)\right| \leqq(1+\delta)\left|a_{11}(x)\right|<$ $\left|a_{12}(x)-\left[a_{11}(x)-a_{22}(x)\right]\right|$. The argument now splits into two cases. 
Case I. For every $x \in \mathscr{Y} ;\left|a_{12}(x)\right| \geqq\left|a_{11}(x)\right|$. In this case we define an invertible $S=\left(s_{i j}\right) \in M_{n}(\mathscr{Y})$ to be the direct sum of the $2 \times 2$ matrix $\left(s_{i j}: i, j \leqq 2\right)$ and the identity element of $M_{n-2}\left(\mathscr{V}^{-}\right)$, where for $x \in \mathscr{V}, s_{11}(x)=s_{22}(x)=1, s_{12}(x)=0$, and $s_{21}(x)=a_{11}(x) / a_{12}(x)$. An easy calculation shows that $\|S\|,\left\|S^{-1}\right\| \leqq 4$, and another calculation shows that for $x \in \mathscr{V}$, the matrix $S U A U^{*} S^{-1}(x)$ has a zero in the upper left hand corner. The existence of $y^{\prime}$ thus contradicts the maximality of the collection $\left\{\mathscr{V}_{i}\right\}_{i \in I}$, and we proceed to

Case II. There is a compact open subset ' $\mathscr{V} \subset \mathscr{V}$ ' such that for $x \in \mathscr{Y},\left|a_{12}(x)\right|<\left|a_{11}(x)\right|$. As before we define an invertible $S=$ $\left(s_{i j}\right) \in M_{n}(\mathscr{W})$ to be the direct sum of the $2 \times 2$ matrix $\left(s_{i j}: i, j \leqq 2\right)$ and the identity element of $M_{n-2}(\mathscr{W})$. This time for $x \in \mathscr{W}$ we take $s_{11}(x)=s_{12}(x)=s_{21}(x)=\left[a_{11}(x) /\left\{a_{12}(x)-\left[a_{11}(x)-a_{22}(x)\right]\right\}\right]^{1 / 2}$ and $s_{22}(x)=$ $s_{11}(x)\left[\left\{a_{12}(x)+a_{22}(x)\right\} / a_{11}(x)\right]$, where the exponent $1 / 2$ denotes any square root taken in such a way that $s_{11} \in C(\mathscr{W})$. (Theorem 1 of [3] enables us to take continuous square roots.) As a result of the inequalities which are valid on $\mathscr{W}$, one has $\left|s_{11}(x)\right|<1$ and $\left|s_{22}(x)\right| \leqq 2+\delta$ for each $x \in \mathscr{W}$; furthermore, $s_{11} s_{22}-s_{12} s_{21} \equiv 1$ on $\mathscr{W}$, and it follows that $\|S\|,\left\|S^{-1}\right\| \leqq 6$. Calculation shows that for $x \in \mathscr{W}, S U A U^{*} S^{-1}(x)$ has a zero for its upper left hand entry, and thus the proof is complete.

The following corollary follows easily by induction on $n$, and we omit its proof.

CoRollary 4.2. If $A \in M_{n}(\mathfrak{X})$ is such that $\sigma[A(x)] \equiv 0$, then there is an invertible $S \in M_{n}(\mathfrak{X})$ such that $S A S^{-1}=\left(a_{i j}\right)$ satisfies $a_{i i} \equiv 0$ for $1 \leqq i \leqq n$.

Proof of Theorem 5. We are given that $\sigma\lfloor A(x)\rfloor \equiv 0$. Choose $S \in M_{n}(\mathfrak{X})$ according to Corollary 4.2 so that $S A S^{-1}=\left(a_{i j}\right)$ satisfies $a_{i i} \equiv 0$ for $1 \leqq i \leqq n$. Define $B_{1}=\left(b_{i j}\right) \in M_{n}(\mathfrak{X})$ by $b_{i i} \equiv i$ for $1 \leqq i \leqq n$ and $b_{i j} \equiv 0$ for $i \neq j$. Also define $C_{1}=\left(c_{i j}\right) \in M_{n}(\mathfrak{X})$ by $c_{i j} \equiv a_{i j} /\left(b_{i i}-b_{j j}\right)$ for $i \neq j$ and $c_{i j} \equiv 0$ for $i=j$. If $B$ and $C$ are defined by $B=S^{-1} C_{1} S$, then it is easy to see that $B_{1} C_{1}-C_{1} B_{1}=S A S^{-1}$, or, what is the same thing, $B C-C B=A$.

\section{REMARKS.}

(1) A stronger version of Lemma 4.1, obtained from the present version by requiring $S$ to be unitary, actually holds. The proof, however, uses a completely different idea and is much longer than the above proof.

(2) A bounded operator $B$ on Hilbert space is called $n$-normal [9] if the $W^{*}$-algebra which $B$ generates satisfies a polynomial identity 
of the form

$$
\sum(\operatorname{sgn} \pi) X_{\pi(1)} X_{\pi(2)} \cdots X_{\pi(2 n)}=0,
$$

where the sum is taken over all permutations $\pi$ on $2 n$ objects. It is known that such a $W^{*}$-algebra is a finite direct sum of algebras each of which has a faithful $C^{*}$-representation as some $M_{k}\left(\mathfrak{X}_{k}\right)$ with $\mathfrak{X}_{k}$ Stonian and $k \leqq n$. Furthermore such a $W^{*}$-algebra has a well-behaved centervalued trace function, so that Theorem 5 can be paraphrased: Any $n$-normal operator with trace zero is the commutator of a pair of $n$-normal operators.

(3) There are at least two classes of operators on Hilbert space which possess well-behaved numerical traces. These are operators in the trace-class [13], and operators in $W^{*}$-algebras which are factors of type $I I_{1}$. Is it true that every operator with trace zero in one of these classes is a commutator?

5. Two examples. In this section we set forth two examples which show that Theorem 2 of [3] and Theorems 1 and 2 of the present paper cannot be extended to the setting in which $\mathfrak{X}$ is assumed only to be a compact Hausdorff, totally disconnected space. In these examples we take $\mathscr{T}$ to be the compact Hausdorff, totally disconnected space consisting of the set $\left\{a_{1}, a_{2}, \cdots, a_{n}, \cdots, 0\right\}$ with the relative topology, where the real sequence $\left\{a_{n}\right\}$ is strictly decreasing to zero and satisfies $\cos \left(1 / a_{n}\right)=\sin \left(1 / a_{n}\right)=1 / \sqrt{2}$ for $n$ odd and $\cos \left(1 / a_{n}\right)=1, \sin \left(1 / a_{n}\right)=0$ for $n$ even.

Example 1. (This example is essentially due to Rellich [11].) Define $A \in M_{2}(\mathscr{T})$ by

$$
\begin{gathered}
A\left(a_{n}\right)=\left(\begin{array}{cc}
1-a_{n} \cos \left(2 / a_{n}\right) & -a_{n} \sin \left(2 / a_{n}\right) \\
-a_{n} \sin \left(2 / a_{n}\right) & 1+a_{n} \cos \left(2 / a_{n}\right)
\end{array}\right) ; \\
A(0)=\left(\begin{array}{ll}
1 & 0 \\
0 & 1
\end{array}\right) .
\end{gathered}
$$

Then, even though $A$ is Hermitian, there exists no unitary $U \in M_{2}(\mathscr{T})$ such that $U A U^{*}(t)$ is in upper triangular form for each $t \in \mathscr{T}$.

Proof. Assume that such a $U=\left(u_{i j}\right)$ exists, and let $U A U^{*}(t)=$ $\left(b_{i j}(t)\right)$. Then the $b_{i j} \in C(\mathscr{T})$, and the vector $\left(\bar{u}_{11}(t), \bar{u}_{12}(t)\right)=V(t)$ has length one at each $t \in \mathscr{T}$ and has entries which are elements of $C(\mathscr{T})$. Futhermore, it is easy to see that $\left[\mathrm{A}(t)-b_{11}(t) I\right] V(t) \equiv 0$. In other words, the vector $V(t)$ is a continuous eigenvector for $A(t)$ cor- 
responding to the eigenvalue $b_{11}(t)$. An easy calculation shows that the eigenvalues of $A\left(a_{n}\right)$ are $1-a_{n}$ and $1+a_{n}$, so that for each $n$, $b_{11}\left(a_{n}\right)=1-a_{n}$ or $b_{11}\left(a_{n}\right)=1+a_{n}$. Furthermore, it is easy to see that the vector $\left(\cos \left(1 / a_{n}\right), \sin \left(1 / a_{n}\right)\right)$ is an eigenvector for $A\left(a_{n}\right)$ corresponding to the eigenvalue $1-a_{n}$, and the vector $\left(\sin \left(1 / a_{n}\right),-\cos \left(1 / a_{n}\right)\right)$ is an eigenvector for $A\left(a_{n}\right)$ corresponding to the eigenvalue $1+a_{n}$. It follows that for $n$ odd, we must have $\left|\bar{u}_{11}\left(a_{n}\right)\right|=1 / \sqrt{2}$, and for $n$ even, we must have $\left|\bar{u}_{11}\left(a_{n}\right)\right|=0$ or 1 . This contradicts $u_{11} \in C(\mathscr{T})$, and completes the proof.

Example 2. Define $A, B \in M_{2}(\mathscr{T})$ by $A(0)=B(0)=0$ and

$$
A\left(a_{n}\right)=\left(\begin{array}{cc}
0 & a_{n} \\
0 & 0
\end{array}\right), \quad B\left(a_{n}\right)=\left(\begin{array}{cc}
0 & (-1)^{n} a_{n} \\
0 & 0
\end{array}\right) .
$$

Then $A(t)$ is unitarily equivalent to $B(t)$ for each $t \in \mathscr{T}$, but there exists no invertible $S \in M_{2}(\mathscr{T})$ such that $S A S^{-1}=B$.

Proof. Suppose such an invertible $S=\left(s_{i j}\right) \in M_{2}(\mathscr{T})$ does exist. Then $S A=B S$, and calculation shows that $s_{21} \equiv 0$. Furthermore, $s_{11}\left(a_{n}\right)=(-1)^{n} s_{22}\left(a_{n}\right)$ for each $n$, and since $S$ is invertible and $s_{21} \equiv 0$, $s_{11}$ and $s_{22}$ are bounded away from zero. It follows that $s_{11}$ and $s_{22}$ cannot both be continuous at zero, a contradiction.

REMARK. While the theory of elements $A \in M_{n}(\mathfrak{X})$ is not very satisfactory for $\mathfrak{X}$ only totally disconnected, it is nevertheless true that $A$ has continuous eigenvalues [4].

\section{BIBLIOGRAPHY}

1. A. Brown, Entire functions on Banach algebras, Michigan Math. J., 10 (1963), 91-96.

2. C. Caratheodory, Theory of Functions vols. I and II, Chelsea, New York (1954).

3. D. Deckard and C. Pearcy, On matrices over the ring of continuous complex-valued functions on a Stonian space, Proc. Amer. Math. Soc., 14 (1963), 322-328.

4. - On algebraic closure in function algebras, Proc. Amer. Math. Soc., 15 (1964), 259-263.

5. N. Dunford, Spectral theory I. Convergence to projections, Trans. Amer. Math. Soc., 54 (1943), 185-217.

6. P. R. Halmos, Finite Dimensional Vector Spaces, Princeton, Van Nostrand (1958).

7. I. Kaplansky, Algebras of type I, Ann. of Math., 56 (1952), 460-472.

8. S. Kurepa, , to appear in Publications de 1'Inst. Math., Srpska Akad., Nauk, Belgrad.

9. C. Pearcy, A complete set of unitary invariants for operators generating finite $W^{*}$-algebras of type I, Pacific J. Math., 12 (1962), 1405-1416.

10. - On unitary equivalence of matrices over the ring of continuous complexvalued functions on a Stonian space, Canad. J. Math., 15 (1963), 323-331.

11. F. Rellich, Perturbation theory of eigenvalue problems, lecture notes, New York University (1953). 
12. C. Rickart, General theory of Banach algebras, Princeton, Van Nostrand (1960).

13. R. Schatten, Norm ideals of completely continuous operators, Berlin, Springer-Verlag (1960).

14. M. H. Stone, Boundedness properties in function-lattices, Canad. J. Math., 1 (1949), 176-186.

15. T. Yen, Trace on $A W^{*}$-algebras, Duke J. Math., 22 (1955), 207-222.

Humble OIL \& ReFining Company

Houston, Texas 



\section{PACIFIC JOURNAL OF MATHEMATICS}

\section{EDITORS}

\author{
Robert Osserman \\ Stanford University \\ Stanford, California
M. G. Arsove
University of Washington
Seattle 5 , Washington

\author{
J. DugundJI \\ University of Southern California \\ Los Angeles 7, California
}

Lowell J. Paige

University of California

Los Angeles 24, California

\section{ASSOCIATE EDITORS}
E. F. BECKENBACH
B. H. NeumanN
F. WOLF
K. YOSIDA

\section{SUPPORTING INSTITUTIONS}

\author{
UNIVERSITY OF BRITISH COLUMBIA \\ CALIFORNIA INSTITUTE OF TECHNOLOGY \\ UNIVERSITY OF CALIFORNIA \\ MONTANA STATE UNIVERSITY \\ UNIVERSITY OF NEVADA \\ NEW MEXICO STATE UNIVERSITY \\ OREGON STATE UNIVERSITY \\ UNIVERSITY OF OREGON \\ OSAKA UNIVERSITY \\ UNIVERSITY OF SOUTHERN CALIFORNIA
}

\author{
STANFORD UNIVERSITY \\ UNIVERSITY OF TOKYO \\ UNIVERSITY OF UTAH \\ WASHINGTON STATE UNIVERSITY \\ UNIVERSITY OF WASHINGTON \\ * * * * \\ AMERICAN MATHEMATICAL SOCIETY \\ CALIFORNIA RESEARCH CORPORATION \\ SPACE TECHNOLOGY LABORATORIES \\ NAVAL ORDNANCE TEST STATION
}

Mathematical papers intended for publication in the Pacific Journal of Mathematics should by typewritten (double spaced), and on submission, must be accompanied by a separate author's résumé. Manuscripts may be sent to any one of the four editors. All other communications to the editors should be addressed to the managing editor, L. J. Paige at the University of California, Los Angeles 24, California.

50 reprints per author of each article are furnished free of charge; additional copies may be obtained at cost in multiples of 50 .

The Pacific Journal of Mathematics is published quarterly, in March, June, September, and December. Effective with Volume 13 the price per volume (4 numbers) is $\$ 18.00$; single issues, $\$ 5.00$. Special price for current issues to individual faculty members of supporting institutions and to individual members of the American Mathematical Society: $\$ 8.00$ per volume; single issues $\$ 2.50$. Back numbers are available.

Subscriptions, orders for back numbers, and changes of address should be sent to Pacific Journal of Mathematics, 103 Highland Boulevard, Berkeley 8, California.

Printed at Kokusai Bunken Insatsusha (International Academic Printing Co., Ltd.), No. 6, 2-chome, Fujimi-cho, Chiyoda-ku, Tokyo, Japan.

PUBLISHED BY PACIFIC JOURNAL OF MATHEMATICS, A NON-PROFIT CORPORATION

The Supporting Institutions listed above contribute to the cost of publication of this Journal, but they are not owners or publishers and have no responsibility for its content or policies. 


\section{Pacific Journal of Mathematics}

\section{Vol. 14, No. $3 \quad$ July, 1964}

Erik Balslev and Theodore William Gamelin, The essential spectrum of a class of ordinary differential operators . . . . . . . . . . . . . . . . . . . .

James Henry Bramble and Lawrence Edward Payne, Bounds for derivatives in

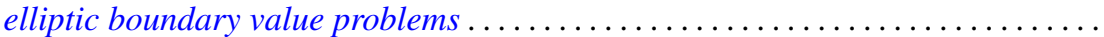

Hugh D. Brunk, Integral inequalities for functions with nondecreasing



William Edward Christilles, A result concerning integral binary quadratic

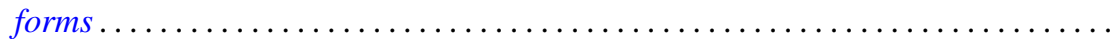

Peter Crawley and Bjarni Jónsson, Refinements for infinite direct decompositions of

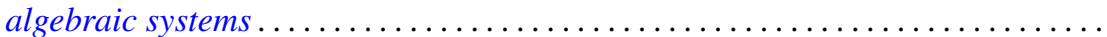

Don Deckard and Carl Mark Pearcy, On continuous matrix-valued functions on a Stonian space.

Raymond Frank Dickman, Leonard Rubin and P. M. Swingle, Another

characterization of the $n$-sphere and related results $\ldots \ldots \ldots \ldots \ldots \ldots$

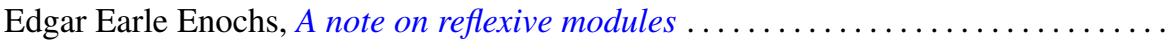

Vladimir Filippenko, On the reflection of harmonic functions and of solutions of the



Derek Joseph Haggard Fuller, Mappings of bounded characteristic into arbitrary

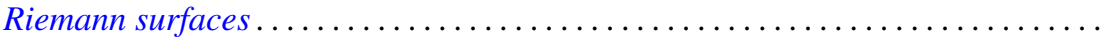
895

Curtis M. Fulton, Clifford vectors . . . . . . . . . . . . . . . . . . . . . . . . . . . . . . 917

Irving Leonard Glicksberg, Maximal algebras and a theorem of Radó . .

919

Kyong Taik Hahn, Minimum problems of Plateau type in the Bergman metric

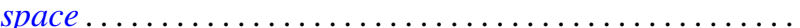

A. Hayes, A representation theory for a class of partially ordered rings...

J. M. C. Joshi, On a generalized Stieltjes trasform

J. M. C. Joshi, Inversion and representation theorems for a generalized Laplace transform ...

Eugene Kay McLachlan, Extremal elements of the convex cone $B_{n}$ of functions ...

Robert Alan Melter, Contributions to Boolean geometry of p-rings ...

James Ronald Retherford, Basic sequences and the Paley-Wiener criterion . . . . . . . 1019

Dallas W. Sasser, Quasi-positive operators. .

Oved Shisha, On the structure of infrapolynomials with prescribed coefficients ..

Oved Shisha and Gerald Thomas Cargo, On comparable means

Maurice Sion, A characterization of weak ${ }^{*}$ convergence ........

Morton Lincoln Slater and Robert James Thompson, A permanent inequality for

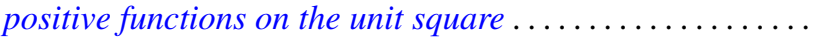

David A. Smith, On fixed points of automorphisms of classical Lie algebras ...

Sherman K. Stein, Homogeneous quasigroups ................

J. L. Walsh and Oved Shisha, On the location of the zeros of some infrapolynomials with prescribed coefficients .

Ronson Joseph Warne, Homomorphisms of $d$-simple inverse semigroups with identity . 